\title{
EFICÁCIA DO SISTEMA DE ESTADIAMENTO DE ENNEKING NO TRATAMENTO DOS TUMORES ÓSSEOS BENIGNOS E LESÕES ÓSSEAS PSEUDOTUMORAIS
}

\author{
EFFICACY OF THE ENNEKING STAGING SYSTEM IN THE TREATMENT \\ OF BENIGN BONE TUMORS AND TUMOR-LIKE BONE LESIONS
}

José Marcos Nogueira Drumond

\section{RESUMO}

Objetivo: Avaliar a eficácia do Sistema de Estadiamento de Enneking (SEE) em determinar o prognóstico, planejar o tratamento cirúrgico e indicar a terapia adjuvante no tratamento de tumores ósseos benignos (TOB) e lesões ósseas pseudotumorais (LOP). Métodos: Foi realizado estudo retrospectivo multicêntrico, descritivo, não randomizado, com amostra representativa de uma grande série de 165 pacientes portadores de 168 tumores ósseos benignos e lesões ósseas pseudotumorais. A casuística foi típica e concordou com a literatura em todos os aspectos. Todos os pacientes foram estadiados no Sistema de Estadiamento de Enneking e fez-se a correlação do estadiamento inicial de cada lesão com o seu comportamento após tratamento conservador ou cirúrgico para determinar a eficácia do SEE. Os devidos tratamentos e as complicações são descritos e analisados. Resultados: O resultado do tratamento mostrou proporção concordante de 95,2\% com o SEE, com intervalo de $95 \%$ de confiança de 90,8 a 97,9\%. De 168 tumores tratados, somente oito (4,8\%) não foram controlados com o tratamento inicial indicado pelo SEE. Há predominância de tumores estadiados como ativos, com 73,2\% das lesões. As recidivas foram mais frequentes no estágio agressivo, sendo estatisticamente significativo $(p<0,001)$. Todos os pacientes estadiados como latentes evoluíram para cura. O cimento ósseo (PMMA) mostrou-se um adjuvante efetivo quando associado a uma cirurgia marginal. $\mathrm{O}$ estudo sugeriu que a cirurgia com margem ampla, para lesões agressivas, poderia controlar melhor a lesão, com menor taxa de recidiva $(p>0,001)$. Para lesões latentes e ativas comprovou-se a eficácia dos tratamentos expectante e de excisão simples ou associada com enxerto ósseo autógeno. Conclusão: Os resultados confirmaram que o SEE foi muito eficaz em determinar o prognóstico, planejar a cirurgia e indicar a terapia adjuvante no tratamento de TOB e LOP.

Descritores - Doenças ósseas; Neoplasias ósseas/diagnóstico/ epidemiologia/patologia/radiografia/cirurgia; Estadiamento de neoplasias

\section{ABSTRACT}

Objective: To evaluate the efficacy of the Enneking Staging System in determining the prognosis, planning surgical treatment and indicating adjuvant therapy for the treatment of benign bone tumors (BBT) and tumor-like bone lesions (TBL). Methods: A retrospective multicentric, descriptive, nonrandomized study was carried out comprising a large series of 165 patients with a total of 168 benign bone tumors and pseudotumoral bone lesions. The patient sampling was typical, and matched the literature in all aspects. All the patients were classified according to the Enneking Staging System, and were reevaluated after either conservative or surgical treatment, to establish the efficacy of the system. Treatment options and clinical complications were analyzed. Results: The results of the treatment provided 95.2\% agreement with the Enneking Staging System, with a 95\% confidence interval of between 90.8 and $97.9 \%$. Of the 168 tumors treated, only eight (4.8\%) could not be controlled by the initial treatment indicated by the Enneking Staging System. Tumors classified as active were the most prevalent, comprising $73.2 \%$ of the lesions. Tumor recurrence was significantly higher $(p<0.001)$ for the aggressive stage. The study also suggested that wide surgical margins could be more efficient in reducing the recurrence of aggressive lesions ( $p>0.001)$. All the patients staged as latent were healed. Bone cement (PMMA) was an effective adjuvant when associated with marginal surgery. For latent and active lesions, the study demonstrated the efficacy of treatments with observation only, or with excision, with or without autogenous bone graft. Conclusion: The results confirm that the Enneking Staging System was very efficient in determining prognosis, assisting surgical planning and indicating adjuvant therapy in the treatment of BBT and TBL.

Keywords - Bone diseases; Bone neoplasms/diagnosis/epidemiology/ pathology/radiography/surgery; Neoplasm staging

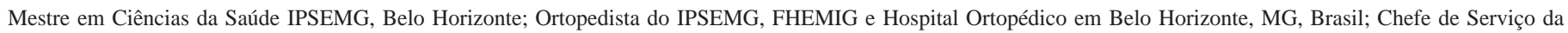
FHEMIG - Fundação Hospitalar do Estado de Minas Gerais.

Trabalho realizado no IPSEMG - Instituto de Previdência dos Servidores do Estado de Minas Gerais, Belo Horizonte

Correspondência: Rua Prof. Estevão Pinto, 555/304, Serra - 30220-060 - Belo Horizonte, MG. E-mail: jmnd@uai.com.br

Declaramos inexistência de conflito de interesses neste artigo 


\section{INTRODUÇÃO}

Os tumores ósseos benignos (TOB) e as lesões ósseas pseudotumorais (LOP) são doenças relativamente raras que acometem principalmente crianças e adultos jovens, com o esqueleto em formação, podendo causar fraturas, deformidades ósseas e articulares, distúrbios da marcha, deixando, às vezes, sequelas incapacitantes por toda a vida.

Feito o diagnóstico do tumor, este deve ser estadiado visando seu tratamento. Enneking foi o primeiro a descrever um sistema para o estadiamento de tumores ósseos benignos e esse sistema é o mais comumente usado $^{(1-5)}$.

Esse sistema de estadiamento introduziu uma linguagem única para avaliar e comparar resultados de diferentes protocolos e séries de pacientes, de diversos centros de tratamento.

Articulando-se o estágio do tumor com as margens cirúrgicas consegue-se o procedimento cirúrgico indicado para se obter o controle e cura da lesão ${ }^{(3,4)}$.

O Sistema de Estadiamento de Enneking (SEE) foi publicado na década de $80^{(1-5)}$ e trabalhos são necessários para testá-lo, comprovar sua eficácia e aperfeiçoálo ${ }^{(6)}$ (Tabela 1).

O objetivo deste trabalho foi avaliar a eficácia do Sistema de Estadiamento de Enneking em determinar o prognóstico, planejar o tratamento cirúrgico e indicar a terapia adjuvante no manejo de tumores ósseos benignos e lesões ósseas pseudotumorais.

\section{MÉTODOS}

Realizou-se estudo observacional descritivo de grande série de casos, não randomizado, retrospectivo e multicêntrico. Os dados foram levantados pelo autor em prontuários e exames de imagens de pacientes tratados no Hospital do IPSEMG (HGIP), Hospital OrtopédicoAMR (HO), Hospital Belo Horizonte (HBH) e Hospital São Francisco de Assis (HSF), todos em Belo Horizonte/ MG. Os exames de imagens foram radiografias simples, tomografia computadorizada, ressonância nuclear magnética, cintilografia e arteriografia. A casuística foi formada por 165 pacientes, com 168 lesões tumorais, submetidos a tratamento conservador ambulatorial ou cirúrgico hospitalar (Tabelas 2 e 3).

Um paciente com tumor de células gigantes (TCG) multicêntrico apresentava quatro lesões. Entre os pacientes com osteocondromas há quatro casos de osteo-
Tabela 1 - Sistema de Estadiamento de Enneking: articulação dos estágios com margens cirúrgicas

\begin{tabular}{c|c|c|c}
\hline $\begin{array}{c}\text { Estágio do tumor } \\
\text { (benigno) }\end{array}$ & $\begin{array}{c}\text { Grau, localização, } \\
\text { metástases }\end{array}$ & $\begin{array}{c}\text { Evolução } \\
\text { clínica }\end{array}$ & Margem para controle \\
\hline 1 & GoToMo & Latente & Intracapsular \\
\hline 2 & GoToMo & Ativo & $\begin{array}{c}\text { Marginal ou intracapsular } \\
\text { mais adjuvante efetivo }\end{array}$ \\
\hline 3 & GoT $_{1-2} \mathrm{M}_{0-1}$ & Agressivo & $\begin{array}{c}\text { Ampla ou marginal mais } \\
\text { adjuvante efetivo }\end{array}$ \\
\hline
\end{tabular}

Go $=$ Benigno, To $=$ Intracapsular, T1 $=$ Extracapsular, Intracompartimental, T2 $=$ Extracapsular, Extracompartimental, $\mathrm{Mo}=$ Ausência de metástases $\mathrm{M} 1=$ Presença de metástases.

Tabela 2 - Tumores ósseos benignos

\begin{tabular}{l|c}
\hline & Número de pacientes \\
\hline Osteocondroma & 31 \\
\hline Encondroma & 14 \\
\hline Tumor de células gigantes & 13 \\
\hline Condroblastoma & 12 \\
\hline Osteoma osteoide & 10 \\
\hline Fibroma condro-mixoide & 2 \\
\hline Lipoma intraósseo & 2 \\
\hline Fibro-histiocitoma benigno & 1 \\
\hline Total & 85 \\
\hline Fonte : SAME HGIP, HO, HBH, HSF, 2008. &
\end{tabular}

Tabela 3 - Lesões ósseas pseudotumorais

\begin{tabular}{l|c}
\hline & Número de pacientes \\
\hline Fibroma não-ossificante & 25 \\
\hline Cisto ósseo simples & 19 \\
\hline Displasia fibrosa & 17 \\
\hline Cisto ósseo aneurismático & 10 \\
\hline Miosite ossificante & 6 \\
\hline Hiperparatireoidismo & 3 \\
\hline Total & 80 \\
\hline
\end{tabular}

Fonte: SAME HGIP, HO, HBH, HSF, 2008.

condromatose. Apenas um paciente, com TCG, apresentou metástases pulmonares. São pacientes consecutivos tratados pelo autor no período de tempo de 16 anos, entre janeiro de 1988, data em que se iniciou a utilização do Sistema de Estadiamento de Enneking (SEE), e janeiro de 2004, exigindo-se um seguimento mínimo de três anos como critério para inclusão dos pacientes.

A média de idade dos pacientes foi de 23,2 anos, sendo a menor com três anos, em um paciente com osteoma osteoide, e a maior com 69 anos, em portador 
de encondroma. São 82 pacientes do sexo masculino e 83 do feminino. O fêmur foi o osso com maior acometimento, com $40 \%$ das lesões, seguido pela tíbia, com 20\%. Outros ossos mais afetados foram: o úmero, a fíbula, o ilíaco e o rádio. O seguimento máximo foi de 19 anos e o mínimo de três anos, sendo a média de 8,35 anos.

Por estágios, 36 pacientes são latentes (B1), 123 são ativos (B2) e nove são agressivos (B3). Entre os agressivos, cinco são tumores de células gigantes (TCG) e quatro são cistos ósseos aneurismáticos (COA).

Foram tratados conservadoramente 39 tumores e o tratamento cirúrgico foi instituído em 129 tumores. A terapia adjuvante foi usada em 30 tumores, utilizando-se a cimentação com polimetilmetacrilato (PMMA) em 29 casos e a radioterapia em um caso.

O tratamento conservador consistiu de observação clínico-radiológica em 29 casos e imobilização gessada para fraturas em dez casos. O tratamento cirúrgico seguiu a articulação dos estágios com as margens cirúrgicas do SEE (Tabela 1). Como métodos de tratamento tivemos os descritos na Tabela 4 e as margens cirúrgicas utilizadas estão descritas na Tabela 5.

Tabela 4 - Métodos Cirúrgicos de Tratamento

\begin{tabular}{l|c}
\multicolumn{1}{c|}{ Tipo } & Número de Casos \\
\hline Excisão simples & 40 \\
\hline Excisão e enxerto ósseo & 31 \\
\hline Excisão e cimentação & 29 \\
\hline Biópsia isolada & 8 \\
\hline Infiltração com corticoide & 8 \\
\hline Osteossíntese & 7 \\
\hline Prótese articular & 5 \\
\hline Excisão segmentar & 3 \\
\hline Artrodese & 1 \\
\hline Total & 132 \\
\hline Fonte: SAME HGIP, HO, HBH, HSF, 2008. &
\end{tabular}

Fonte: SAME HGIP, HO, HBH, HSF, 2008.

Tabela 5 - Margens Cirúrgicas Utilizadas

\begin{tabular}{c|c}
\hline Tipo & Número de Casos \\
\hline Intracapsular & 53 \\
\hline Marginal & 71 \\
\hline Ampla & 5 \\
\hline Total & 129 \\
\hline Fonte: SAME HGIP, HO, HBH, HSF, 2008.
\end{tabular}

A ampliação da margem com broca rotatória de alta velocidade foi realizada em todos os casos de excisão intralesional, como também a eletrocauterização da cavidade. Em seis casos foram inseridos fios de Kirschner múltiplos dentro da cimentação. Enxerto ósseo ilíaco autógeno foi utilizado em 29 casos e enxerto homólogo em dois casos. Fez-se deslizamento ósseo em um caso. Um paciente com COA de pelve foi tratado com embolização arterial seletiva e radioterapia. Foram realizados exames anatomopatológicos em todos os pacientes submetidos a tratamento aberto.

Todos os pacientes foram estadiados pelo SEE e identificaram-se o tratamento realizado, margem cirúrgica obtida, se foi usado algum adjuvante. Determinados ainda a data de início do tratamento, seguimento ambulatorial mínimo de três anos, resultado do tratamento e presença de recidivas e cura. Posteriormente, fez-se a correlação do estadiamento inicial de cada lesão com o seu comportamento no seguimento para determinar a eficácia do SEE para prever o prognóstico, planejar o tratamento cirúrgico e indicar a terapia adjuvante.

O critério para determinação da cura foi o quadro clínico-radiológico após três anos de seguimento e para constatação de recidiva foi o exame de imagem ou o exame anatomopatológico após a reoperação.

Para uma população considerada grande exige-se uma amostra de 141 pacientes. Com uma amostra de 165 indivíduos, efeito médio, nível de significância de $5 \%$ e dois graus de liberdade, o poder desse estudo é de $94 \%$. Com apenas um grau de liberdade, o poder é de $97 \%$.

$\mathrm{Na}$ análise estatística foram utilizados o teste de qui-quadrado, o teste exato de Fisher e o cálculo dos intervalos de confiança para os percentuais de concordância. Em todas as análises considerou-se o nível de significância de 5\% ou 0,05.

Foi realizada revisão bibliográfica nas bases de dados Medline e Lilacs em um período de 10 anos, entre janeiro de 1997 e dezembro de 2006. Foram encontrados 450 resumos sobre o tema, sendo selecionados 100 trabalhos com texto completo, que apresentavam melhores metodologias e níveis de evidências científicas, além dos trabalhos clássicos sobre a matéria, independente da data de publicação.

O presente projeto foi aprovado pelo Conselho de Ética em Pesquisa do Hospital do IPSEMG (HGIP). 


\section{RESULTADOS}

O resultado do tratamento mostrou alto percentual de concordância com o Sistema de Estadiamento de Enneking (SEE) nos três parâmetros de determinação do prognóstico, do planejamento cirúrgico e da terapia adjuvante (Tabela 6).

Tabela 6 - Concordância entre os resultados do tratamento e o Sistema de Estadiamento de Enneking (SEE) com relação ao prognóstico, planejamento cirúrgico e terapia adjuvante.

\begin{tabular}{c|c|c|c|c|c|c}
\hline \multirow{2}{*}{ SEE } & \multicolumn{2}{|c|}{ Prognóstico } & \multicolumn{2}{c|}{ Planejamento cirúrgico } & \multicolumn{2}{c}{ Terapia adjuvante } \\
\cline { 2 - 7 } & $\mathbf{n}(\mathbf{1})$ & $\%$ & $\mathbf{n}$ & $\%$ & $\mathbf{n}$ & $\%$ \\
\hline Sim & 160 & $95,2 \%$ & 121 & $93,8 \%$ & 26 & $86,7 \%$ \\
\hline Não & 8 & $4,8 \%$ & 8 & $6,2 \%$ & 4 & $13,3 \%$ \\
\hline Total & 168 & $100,0 \%$ & 129 & $100,0 \%$ & 30 & $100,0 \%$ \\
\hline (1) $\mathrm{n}=$ Número de casos
\end{tabular}

A análise global, abrangendo todos os tumores, mostrou uma proporção concordante de $95,2 \%$ entre o tratamento e o SEE, com um intervalo de $95 \%$ de confiança para essa proporção de 90,8 a 97,9\% (Tabela 7 e Figura 1).

Tabela 7 - Concordância entre os resultados do tratamento de Tumores ósseos benignos e Lesões ósseas pseudotumorais e o Sistema de Estadiamento de Enneking (SEE)

\begin{tabular}{c|c|c|c|c|c|c}
\hline \multirow{2}{*}{ SEE } & \multicolumn{2}{|c|}{$\begin{array}{c}\text { Tumores ósseos } \\
\text { benignos }\end{array}$} & \multicolumn{2}{c|}{$\begin{array}{c}\text { Lesões ósseas } \\
\text { pseudotumorais }\end{array}$} & \multicolumn{2}{|c}{ Total } \\
\cline { 2 - 7 } & $\mathbf{n}(\mathbf{1})$ & $\%$ & $\mathbf{n}$ & $\%$ & $\mathbf{n}$ & $\%$ \\
\hline Sim & 85 & $96,6 \%$ & 75 & $93,8 \%$ & 160 & $95,2 \%$ \\
\hline Não & 3 & $3,4 \%$ & 5 & $6,2 \%$ & 8 & $4,8 \%$ \\
\hline Total & 88 & $100,0 \%$ & 80 & $100,0 \%$ & 168 & $100,0 \%$ \\
\hline (1) $\mathrm{n}=$ Número de Casos
\end{tabular}

\begin{tabular}{l|c|c}
\hline \multicolumn{1}{c|}{ Grupo } & $\begin{array}{c}\text { Proporção } \\
\text { concordante }\end{array}$ & $\begin{array}{c}\text { Intervalo de 95\% de confiança } \\
\text { para a proporção (\%) }\end{array}$ \\
\hline Tumores ósseos benignos & 96,6 & $90,4-99,3$ \\
\hline Lesões ósseas pseudotumorais & 93,8 & $86,0-97,9$ \\
\hline Total & 95,2 & $90,8-97,9$ \\
\hline
\end{tabular}

Obs.: Por definição o intervalo de confiança para uma proporção não é simétrico em torno do valor estimado para a proporção, como ocorre no caso de intervalo de confiança para a média

O resultado final do tratamento mostrou que, dos 168 tumores tratados, somente oito $(4,8 \%)$ não foram controlados com o tratamento inicial indicado pelo SEE

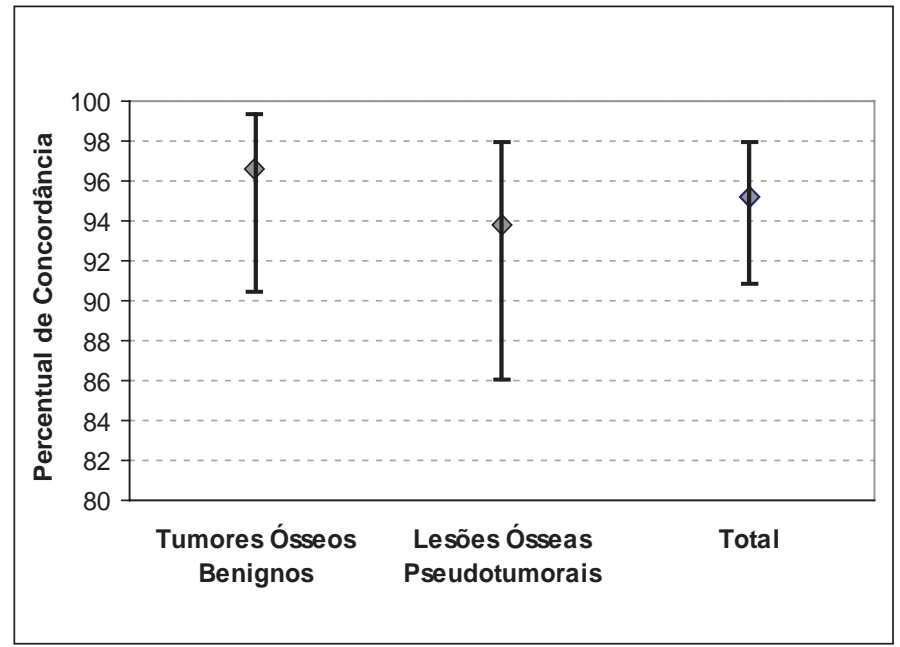

Figura 1 - Intervalos de $95 \%$ de confiança para o percentual estimado de concordância em cada grupo

(Tabela 7). Clinicamente, de 165 pacientes tratados, 159 ficaram curados ou assintomáticos, quatro estão sintomáticos e dois foram a óbito.

Quando os tumores são divididos por estágios, houve predominância do ativo (B2), com 73,2\% das lesões, ficando o latente (B1) com $21,4 \%$ e o agressivo (B3) com 5,4\%, como apresentado na Tabela 8 . Neste estudo, o cisto ósseo simples foi responsável por mais de $50 \%$ dos diagnósticos feitos devido a uma fratura patológica, seguido pelo fibroma não ossificante e a displasia fibrosa.

Tabela 8 - Concordância por estágios entre os resultados do tratamento e o Sistema de Estadiamento de Enneking (SEE)

\begin{tabular}{c|c|c|c|c|c|c|c|c}
\hline \multirow{2}{*}{ SEE } & \multicolumn{2}{|c|}{ Latente } & \multicolumn{2}{c|}{ Ativo } & \multicolumn{2}{c|}{ Agressivo } & \multicolumn{2}{c}{ Total } \\
\cline { 2 - 9 } & $\mathbf{n}(1)$ & $\%$ & $\mathbf{n}$ & $\%$ & $\mathbf{n}$ & $\%$ & $\mathbf{n}$ & $\%$ \\
\hline Sim & 36 & $100,0 \%$ & 119 & $96,7 \%$ & 5 & $55,6 \%$ & 160 & $95,2 \%$ \\
\hline Não & 0 & $0,0 \%$ & 4 & $3,3 \%$ & 4 & $44,4 \%$ & 8 & $4,8 \%$ \\
\hline Total & 36 & $100,0 \%$ & 123 & $100,0 \%$ & 9 & $100,0 \%$ & 168 & $100,0 \%$ \\
\hline (1) $\mathrm{n}=$ Número de casos
\end{tabular}

Entre os 129 pacientes submetidos à cirurgia tivemos oito recidivas (quatro B2 e quatro B3), como apresentado na Tabela 9, e uma taxa global de 6,2\% (Tabela 6).

As recidivas foram mais frequentes no estágio agressivo (B3) com taxa de $44,4 \%$ (quatro em nove pacientes). Aí se inclui o tumor de células gigantes (TCG) com três recidivas, estadiadas como B3, em 16 tumores tratados, taxa de 18,75\%. Nenhum TCG ativo (B2) reci- 
Tabela 9 - Recidivas

\begin{tabular}{c|c|c|c|c|c|c}
\hline Nome & Tipo & Sexo & Idade & Estadiamento & Cirurgia & Recidiva \\
\hline 1. RDS & TCG & M & 19 & B3 & Marginal (úmero proximal) + adjuvante PMMA & 14 meses \\
\hline 2. DSG & TCG & M & 27 & B3 & Marginal (rádio distal) + adjuvante PMMA & 11 meses \\
\hline 3. GGT & TCG & F & 38 & B3 & Marginal ( fêmur distal) + adjuvante PMMA & 6 meses (malignização) \\
\hline 4. BHOM & COS & M & 5 & B2 & Intracapsular (colo femoral) & 17 meses \\
\hline 5. AJT & DF & F & 23 & B2 & Intracapsular (colo femoral) + enxerto ósseo autógeno & 48 meses \\
\hline 6. ASS & DF & F & 32 & B2 & Intracapsular (diáfise umeral) + enxerto ósseo autógeno & 22 meses \\
\hline 7. CCCR & COA & M & 5 & B3 & Intracapsular (asa ilíaca) + embolização + radioterapia & 60 meses (malignização) \\
\hline 8. RCB & COA & M & 10 & B2 & Intracapsular (fêmur proximal) + enxerto ósseo autógeno & 12 meses \\
\hline
\end{tabular}

Fonte: SAME HGIP, HO, HBH, HSF, 2008.

$\mathrm{PMMA}=$ Polimetilmetacrilato $\mathrm{TCG}=$ Tumor de células gigantes $\mathrm{COS}=$ Cisto ósseo simples $\mathrm{DF}=$ Displasia fibrosa $\mathrm{COA}=$ Cisto ósseo aneurismático

divou. Para pacientes com TCG operados com cirurgia marginal e cimentação, a taxa de recidiva foi de $30 \%$ (três casos B3 em dez casos totais). Esses três pacientes foram reoperados. Um foi submetido a cirurgia ampla e endoprótese de ombro e ficou curado. Outro apresentou uma segunda recidiva em cirurgia marginal com cimentação e, em seguida, uma terceira recidiva e foi perdido do seguimento quando se indicou cirurgia ampliada e substituição com enxerto fibular para tumor de rádio distal. O terceiro apresentou malignização primária para fibrossarcoma de fêmur e foi tratado com endoprótese femoral.

Os quatro tumores B2 que recidivaram, taxa de 3,3\% (quatro em 123 casos), eram portadores de cisto ósseo simples do colo femoral, displasia fibrosa de diáfise de úmero e outra de colo femoral, cisto ósseo aneurismático de colo femoral (Tabelas 8 e 9). Todos foram reoperados e curados com cirurgia intracapsular e enxertia óssea autógena.

A recidiva mais precoce ocorreu após seis meses, a mais tardia após cinco anos, com média de 23,75 meses nos oito casos recidivados (Tabela 9).
Todos os pacientes estadiados como latentes (B1) evoluíram para cura, taxa de 100\% (Tabela 8).

A terapia adjuvante com cimento PMMA apresentou apenas três recidivas (10,3\% ) em 29 casos operados. Os três tumores eram TCG estadiados como B3.

Dos nove pacientes estadiados como $\mathrm{B} 3$, cinco não recidivaram (dois com TCG e três com COA). Desses, quatro foram operados com cirurgia ampla e um com cirurgia marginal. E ainda, três foram submetidos a substituição com prótese, um a enxertia com deslizamento ósseo e outro a cirurgia marginal mais cimentação (Tabela 10).

Um paciente com COA de pelve (B3), tratado com embolização arterial seletiva e radioterapia, apresentou malignização secundária para fibrossarcoma, cinco anos após o tratamento. Os dois tumores que sofreram malignização evoluíram para fibrossarcomas estadiados como II-B. Foram reoperados com cirurgias com margens amplas, endoprótese femoral para TCG de fêmur distal e ressecção pélvica para COA de asa ilíaca. Em seguida, foram tratados com quimioterapia, mas apresentaram metástases pulmonares, evoluindo para óbito.

Tabela 10 - Tumores Agressivos (B3) Não Recidivados

\begin{tabular}{c|c|c|c|c|c}
\hline Nome & Tipo & Sexo & Idade & Estadiamento & Cirurgia \\
\hline 1.VCA & TCG & $\mathrm{F}$ & 15 & $\mathrm{GoT}_{2} \mathrm{Mo}$ & Marginal joelho (tíbia) + cimento ósseo \\
\hline 2. JRA & TCG & $\mathrm{F}$ & 13 & $\mathrm{GoT}_{2} \mathrm{M}_{1}$ & Ampla + endoprótese joelho + toracotomia \\
\hline 3. AJOR & COA & $\mathrm{M}$ & 30 & $\mathrm{GoT}_{2} \mathrm{Mo}$ & Ampla + endoprótese joelho \\
\hline 4. CAMS & COA & $\mathrm{M}$ & 29 & GoT $_{2} \mathrm{Mo}$ & Ampla + prótese total quadril \\
\hline 5. EFA & COA & $\mathrm{M}$ & 17 & GoT $_{2} \mathrm{Mo}$ & Ampla + enxertia e deslizamento ósseo tornozelo (tíbia) \\
\hline
\end{tabular}

Fonte: SAME HGIP, HO, HBH, HSF, 2008. 
O paciente com TCG multicêntrico, com quatro lesões epifisárias no mesmo membro inferior, e outro que apresentou metástases pulmonares por TCG, sendo submetido a toracotomia, ficaram curados.

Como complicações tardias mais comuns tivemos: quatro casos de encurtamento de membro inferior em três pacientes com COA e um com displasia fibrosa, relacionados com deformidade angular e acometimento de placa de crescimento; cinco casos de artrose, sendo um relacionado com deformidade angular pela displasia fibrosa, e quatro casos após cimentação para TCG: no joelho (tíbia proximal, dois casos), na subtalar (tumor de calcâneo) e no punho (tumor de rádio distal). Houve apenas um caso de infecção profunda, tardia, em encondroma de tíbia proximal operado com cimentação, sendo necessário remover o cimento (Tabela 11).

Tabela 11 - Complicações

\begin{tabular}{l|c}
\hline & Número de Casos \\
\hline Artrose tardia & 5 \\
\hline Discrepância de membros & 4 \\
\hline Malignização & 2 \\
\hline Limitação articular & 2 \\
\hline Deformidade em varo & 2 \\
\hline Infecção superficial & 2 \\
\hline Fratura do fêmur & 2 \\
\hline Genuvalgo & 1 \\
\hline Ruptura tendinosa (ELP) & 1 \\
\hline Infecção profunda & 1 \\
\hline Meralgia parestésica & 1 \\
\hline Bursite do quadril & 1 \\
\hline
\end{tabular}

Fonte: SAME HGIP, HO, HBH, HSF, 2008.

\section{DISCUSSÃO}

A casuística deste estudo foi típica e concordou com a literatura em todos os aspectos: tumores mais frequentes, idade e sexo, ossos mais acometidos ${ }^{(7,8)}$. O seguimento mínimo para inclusão no estudo foi de três anos porque essas lesões geralmente apresentam recidiva nos primeiros dois anos após o tratamento ${ }^{(9-11)}$.

Foi observado que houve predominância de lesões situadas no estágio ativo (B2), sendo o cisto ósseo simples o mais diagnosticado através de fraturas. A prática clínica mostra que a grande maioria das lesões benignas que exigem atendimento médico, por ser sintomáticas e provocar fraturas, são benignas ativas ${ }^{(12)}$.
Notou-se ainda que as chances de falha do tratamento cirúrgico e consequente recidiva do tumor aumentam à medida que se caminha dos estágios latente (B1) para ativo (B2) e progressivamente para agressivo (B3), sendo estatisticamente significativo. ( $p<0,001)$ (Tabela 8). O estudo mostrou que o cimento ósseo (PMMA) foi um adjuvante efetivo quando associado a uma cirurgia marginal (Tabela 6). Para os pacientes com TCG operados com cimentação, a taxa de recidiva foi alta, mas se equipara a de outros autores publicados ${ }^{(9,13,14)}$. A recorrência maior entre os tumores estadiados como B3 está de acordo com alguns autores ${ }^{(9,15)}$, embora outros não tenham encontrado resultado semelhante $\mathrm{e}^{(10,16)}$.

Este estudo sugere que a cirurgia com margem ampla para lesões benignas agressivas (B3), por não deixar doença microscópica residual, poderia controlar melhor a lesão, com menor taxa de recorrência, embora, devido à pequena casuística nesse estágio, isso não seja estatisticamente significante ( $\mathrm{p}>0,001)$ (Tabelas 9 e 10). A afirmação concorda com a literatura que relata que cirurgias, inicialmente com margens amplas, reduziriam o risco de recorrência, mas com o custo de perda de função e, por isso, não são preferidas, assumindo-se o risco de eventuais recidivas ${ }^{(6,8,17)}$.

Afora os dois pacientes que malignizaram e um que foi perdido do seguimento, todos os estadiados como B3 ficaram curados, sugerindo a eficácia do tratamento.

A ausência de recidivas em tumores latentes (B1) e a baixa taxa de recidiva para lesões ativas (B2) atestam a eficácia dos tratamentos expectante e de excisão simples ou associada com enxerto ósseo ilíaco autógeno, o que é corroborado pela literatura ${ }^{(7,8)}$.

Uma análise dos oito pacientes que recidivaram mostrou que os dois pacientes que malignizaram provavelmente não seriam controlados por qualquer tipo de tratamento, uma vez que se tratava de fibrossarcomas de alto grau (II-B) ${ }^{(1-5)}$. Um paciente com TCG de rádio distal (B3), que foi perdido do seguimento, poderia ter sido controlado com cirurgia ampla e substituição com enxerto fibular, como atesta a literatura ${ }^{(7,8)}$, embora haja relatos de maior agressividade em TCG de rádio distal $^{(9,10)}$. Quatro pacientes foram curados com reoperação intracapsular com enxertia óssea autógena. Um paciente com TCG de úmero proximal (B3) foi curado com cirurgia ampla e substituição com endoprótese (Tabela 9).

As complicações como malignização primária e secundária à radioterapia $^{(18,19)}$, metástase pulmonar por 
TCG ${ }^{(20)}$ e apresentação multicêntrica do TCG ${ }^{(21)}$ estão bem descritas na literatura. A artrose tardia pós-cimentação não foi encontrada por alguns autores ${ }^{(10,15,22)}$, mas é descrita por outros que sugerem a enxertia óssea subcondral para proteger a cartilagem ${ }^{(22)}$.

O Sistema de Estadiamento de Enneking é um sistema de estadiamento cirúrgico que foi elaborado para servir como guia para o tratamento cirúrgico de tumores musculoesqueléticos. Foi testado e adotado em 1980 pela Musculoskeletal Tumor Society (MSTS) ${ }^{(1,2)}$ e pelo American Joint Committee for Cancer Staging and End Results Reporting (AJCC) ${ }^{(23)}$. O sistema foi criado numa época em que radiografias simples eram o único exame de imagem utilizado para estadiar os pacientes. Até agora, o Sistema de Enneking não foi modificado e permanece inalterado por mais de 25 anos, um tempo de grandes avanços em métodos de imagens, genética, oncologia molecular, terapia adjuvante, enxertia óssea e técnica cirúrgica ${ }^{(24)}$.

Em levantamento da literatura disponível, observase que esse sistema de estadiamento não foi validado estatisticamente em amplos estudos multicêntricos. Em 2002, a AJCC, que utilizava o Sistema de Enneking, fez modificações no estadiamento de sarcomas, subs-

\section{REFERÊNCIAS}

1. Enneking WF, Spanier SS, Goodman MA. A system for the surgical staging of musculoskeletal sarcoma. Clin Orthop Relat Res. 1980;(153):106-20.

2. Enneking WF. Staging of musculoskeletal neoplasms. Musculoskeletal Tumor Society. Skeletal Radiol. 1985;13(3):183-94.

3. Enneking WF. A system of staging musculoskeletal neoplasms. Clin Orthop Relat Res. 1986;(204):9-24.

4. Enneking WF. A system of staging musculoskeletal neoplasms. Instr Course Lect. 1988;37(1):3-10.

5. Garcia Filho RJ. Estadiamento de Enneking dos tumores músculo-esqueléticos. Rev Bras Ortop. 1991; 26(8):275-278.

6. Peabody TD, Gibbs CP Jr, Simon MA. Evaluation and staging of musculoskeletal neoplasms. J Bone Joint Surg Am. 1998;80(8):1204-18.

7. Campanacci M. Bone and soft tissue tumors. New York: SpringerVerlag; 1999

8. Schajowicz F. Neoplasias ósseas e lesões pseudotumorais. Rio de Janeiro: Revinter; 2000.

9. O'Donnell RJ, Springfield DS, Motwani HK, Ready JE, Gebhart MC, Mankin HJ. Recurrence of giant-cell tumors of the long bones after curettage and packing with cement. J Bone Joint Surg Am. 1994;76(8):1827-33.

10. Saiz P, Virkus W, Piasecki P, Templeton A, Shott S, Gitelis S. Results of giant cell tumor of tumor treated with intralesional excision. Clin Orthop Relat Res. 2004;(424):221-6.

11. Gibbs CP, Lewis VO, Peabody T. Beyond bone grafting: techniques in the surgical management of benign bone tumors. Instr Course Lect. 2005;54:497-503.

12. Wolf RE, Enneking WF. The staging and surgery of musculoskeletal neoplasms. Orthop Clin North Am. 1996;27(3):473-81.

13. Nicholson NC, Ramp WK, Kneisl JS, Kaysinger KK. Hydrogen peroxide inhibits giant cell tumor and osteoblast metabolism in vitro. Clin Orthop Relat Res. 1998;(347):250-60.

14. Camargo OP, Oliveira NRB, Campos R, Croci AT. Lesões ósseas benignas tituindo a localização compartimental pelo tamanho do tumor e dividindo em três categorias os pacientes com metástases skip, metástases pulmonares e outras metástases $^{(25,26)}$.

Os resultados confirmaram que o SEE foi muito eficaz em determinar o prognóstico, planejar a cirurgia e indicar a terapia adjuvante no tratamento dos tumores ósseos benignos e lesões ósseas pseudotumorais (Tabelas 6 e 7). Esse sistema é o único existente para lesões ósseas benignas e, até onde sabemos por pesquisa da literatura, não há trabalho publicado para sua validação.

Embora este estudo tenha tido o objetivo de avaliar o sistema e tenha comprovado sua eficácia até a presente data, sugerimos que estudos multi-institucionais, com casuísticas incluindo um grande número de pacientes, sejam realizados para reavaliar o sistema e, eventualmente, atualizá-lo à luz dos novos conhecimentos.

\section{CONCLUSÃO}

O Sistema de Estadiamento de Enneking foi muito eficaz em determinar o prognóstico, planejar a cirurgia e indicar a terapia adjuvante no tratamento dos tumores ósseos benignos e lesões ósseas pseudotumorais.

agressivas e ressecção mais metilmetacrilato: seguimento longo (1974-1989) de 151 casos. Rev Bras Ortop. 1990;25(1):3-6.

15. Oda Y, Miura H, Tsuneyoshi M, Iwamoto Y. Giant cell tumor of bone: oncological and functional results of long-term follow-up. Jpn J Clin Oncol. 1998;28(5):323-8.

16. Ghert MA, Rizzo M, Harrelson JM, Scully SP: Giant-cell tumor of the appendicular skeleton. Clin Orthop Relat Res. 2002;(400):201-10.

17. Gitelis S, Mallin B, Piasecki P, Turner F. Intralesional excision compared with en bloc resection for giant cell tumors of bone. J Bone Joint Surg Am. 1993;75(11):1648-55.

18. Brindley GW, Greene JF Jr, Frankel LS. Case reports: malignant transformation of aneurysmal bone cysts. Clin Orthop Relat Res. 2005;438:282-7.

19. Bertoni F, Bacchini P, Staals EL. Malignancy in giant cell tumor of bone. Cancer. 2003;97(10):2520-9.

20. Cheng JC, Johnston JO. Giant cell tumor of bone. Prognosis and treatment of pulmonary metastases. Clin Orthop Relat Res. 1997;(338):205-1.

21. Hoch B, Inwards C, Sundaram M, Rosenberg AE. Multicentric giant cell tumor of bone. Clinicopathologic analysis of thirty cases. J Bone Joint Surg Am. 2006;88(9):1998-2008.

22. Campanacci M, Capanna R, Fabbri N, Bettelli G. Curettage of giant cell tumor of bone: reconstruction with subchondral grafts and cement. Chir Organi Mov. 1990;75(1 Suppl):212-3.

23. Fleming ID, Cooper JS, Henson DE, editors. AJCC Cancer Staging Manual. 5th ed. Philadelphia:Lippincott-Raven;1997.

24. Heck RK Jr, Peabody TD, Simon MA. Staging of primary malignancies of bone. CA Cancer J Clin. 2006;56(6):366-75.

25. Greene FL, Page DL, Fleming ID, editors. AJCC Cancer Staging Manual. 6th ed. New York: Springer-Verlag; 2002.

26. Heck RK Jr, Stacy GS, Flaherty MJ, Montag AG, Peabody TD, Simon MA. A comparison study of staging systems for bone sarcomas. Clin Orthop Relat Res. 2003;(415):64-71. 